\title{
Improvements in business operations and customer experience through data science and Artificial Intelligence
}

\author{
Cristian-Constantin POPESCU \\ Reputation.com, Redwood City, CA, United States \\ cpopescu@reputation.com
}

\begin{abstract}
Faced with increased competitive pressures from online businesses and rapidly changing consumer behaviors, traditional businesses with an online channel as well as a brick and mortar presence increasingly turn to data science and artificial intelligence to make better decisions and enhance their operations. The changing market dynamics are heavily influenced by the overwhelming adoption of mobile phones and data connections. As a result, consumers are better informed, make more real time purchase decisions, and share their experience through review sites and social media. Businesses leverage this large amount of user generated content by extracting the most useful pieces of information from it with text analytics and machine learning algorithms that identify patterns and consumer sentiment at scale. They use the critical pieces of customer feedback to market their products and services better, attract more customers, and offer them a better experience. Four uncertainties and challenges that these business encounter are the following: (1) how to improve online reputation; (2) discoverability and engagement levels of online traffic; (3) how to measure and improve the customer experience; (4) monitoring and benchmarking against competitors. The direct benefits of using Data Science and Artificial Intelligence to address those challenges are: use of standard metrics to measure strengths and weaknesses in online reputation; understanding patterns of customer behavior; listening to customer feedback at scale and extracting actionable insights directly applicable in improving operations; taking corrective actions to avoid losing customers to competitors. This paper will also analyze the competitive landscape in the field of Data Analytics and Insights solutions based on consumer generated feedback. Additionally, it will illustrate a series of case studies across multiple industries, with the purpose of exposing best practices that can be leveraged by businesses in their decision-making process.
\end{abstract}

Keywords: Online Reputation Management, Artificial Intelligence, Big Data, Text Analytics, Customer Experience, insights, competitive reporting, data-driven decisions.

\section{Introduction}

Traditional ways of finding local businesses through directories such as yellow pages are becoming obsolete. Using mobile phones and powerful search engines, customers have instant access to more information about businesses than ever before: details about product offering, reviews of customer service, geographical location, or contact information. They leverage these data points of online reputation to make purchase decisions, so business find that managing online reputation is becoming more important than ever.

Savvy employees from companies' marketing, customer experience, or operations departments use analytics tools to track customer journeys and make sense of those journeys by correlating them with feedback that customers provide online via review sites, social media, and custom surveys. Companies then use those insights to optimize their customer acquisition strategy and to improve its business operations.

According to the market research firm "Markets and Markets" the Customer Analytics market is estimated to grow from \$1.6Bn in 2014 to north of \$4bn in 2019. 


\section{Challenges that companies deal with}

To grow revenue a company engages with potential customers in an attempt to persuade them to purchase its goods or services. These consumers provide post-purchase feedback, whether in a public form, through review sites or social media, or in private via surveys. The business learns the consumers' preferences from this feedback and uses those learnings to make operational improvements, and, as a result provide a better customer experience. Consequently, the clients it serves are happier and leave better reviews of the businesses online. This improved online reputation leads to a higher visibility of the company on search engines and conveys a higher credibility to prospective customers, which persuades more of them to make a purchase. This virtuous cycle repeats itself.

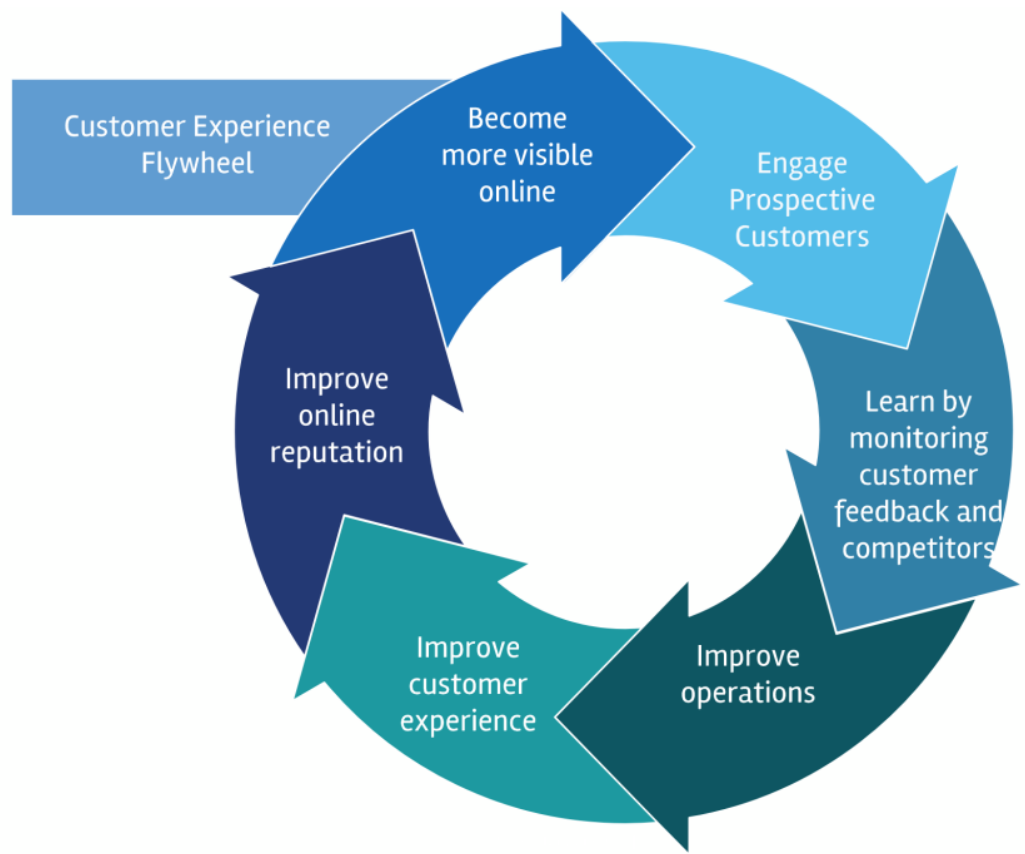

Figure 1. The customer experience flywheel

Source: Author's own research.

To improve every step of the cycle, businesses need insights across several areas:

1. how to improve their online reputation

2. how visible they are and how prospective customers engage with their brands and physical points of retail

3. how to measure and improve their customer experience

4. how to monitor and benchmark against competitors

\section{Improving Online Reputation}

To grow revenue, companies focus on retaining existing customers and acquiring new ones. To do so, they need to have a robust online presence and reputation. But in today's rapidly changing digital environment traditional companies with a physical retail presence need to keep up with all the online environment change in order to stay competitive. It can be challenging to stay appraised which are all the key drivers of online reputation and what makes their retail locations highly visible online. To meet that challenge online reputation 
management software providers have created metrics that inform businesses of their perceived reputation and visibility in the digital realm, such as Binary Fountain's "Provider Social Index" in the Healthcare industry and Reputation.com's "Reputation Score" across multiple industries.

Measuring online visibility and engagement

PICBE | 806

Businesses want to know how people find them online. Typically, they can learn that when consumers click on ads, are referred by third parties to the company's website, visit directly the company's website through direct recall of the brand, or discover the businesses via aggregators such as search engines, social media, blogs, or industry-specific review sites. Marketers are also interested what actions people take when they find these businesses, as well as what is the profile of their users. Traffic insights and analytics tools such as those from Yext, Moz, or Reputation.com help businesses find out their visitors' online traffic pattern at a local, brand, or national level.

\section{Measuring customer experience}

Understanding customer experience is important in order to identify strengths that can be amplified, to pinpoint weaknesses that can be addressed, and to inform strategy. Companies like Medallia, Reputation.com, or Clarabridge are able to leverage text analytics and a branch of artificial intelligence called "machine learning" in enabling their client businesses to extract the key aspects of consumer experience from unstructured data such as online reviews, surveys, or customer communications.

\section{Monitoring competitors}

Managers often need competitive intelligence to make strategic decisions about their business. By benchmarking their brand or physical locations to those of competitors, executives can uncover their business's strengths and weaknesses and use that insight to make better decisions. The solution to monitor competitors is provided via the competitive insights drawn from online reviews and surveys by companies like Reputation.com, Birdeye, or Yext.

\section{Solutions to challenges of improving customer experience Comprehensive benchmarks of Online Reputation}

Online Reputation Management companies have created metrics of online reputation to help their clients keep track of all their key drivers of brand perception among consumers. These drivers range from average star rating on review sites, to review volume and recency, search engine visibility, accuracy of business listings, or engagement of social media pages. These metrics are tailored to each industry and their exact calculation is usually proprietary to the vendor creating them. Businesses use online reputation benchmarks to compare against industry averages and best in class facilities, set strategic targets, rally employees around them, and reward employees upon successfully achieving those targets.

\section{Digital-traffic Insights}

The most common solutions to monitor online traffic are focused around search engine results pages and business listings on review sites. Search engine trackers typically measure rankings in searches across a predefined list of keywords or the visibility of 
businesses in Knowledge Cards or on Maps. Business listings traffic analytics focus around displaying the sources of traffic and the actions that people take when discovering business listings. Companies use such insights to increase the accuracy of their listings across major channels by working with software providers that automatically keep the business listings in sync across hundreds of review sites. Business listings traffic insights can also convey the demographic or geographical profile of visitors, which can help marketers more effectively target their desired customers via advertising or social posting.

\section{Operational Insights}

In today's competitive environment companies with a physical retail presence need to ensure they deliver a top-notch customer experience. Small businesses can read the online reviews that their customers leave after completing a transaction, but that as the review volume scales, it can become difficult and time consuming to identify the key drivers of customer satisfaction. Customer analytics companies use Big Data as well as text mining and analytics to pinpoint the trends, patterns, and main topics that customers communicate about on digital channels. These techniques also uncover the sentiment associated with consumer-to-business communications. Typical such communications consist of online feedback via review sites, surveys, social media, or direct messaging. While rudimentary text analytics can use just keywords or rules to identify topics, the accuracy of text analytics is significantly enhanced by using machine learning algorithms.

A common type of report delivered in this area is the word cloud that uses a visual display of words or phrases that are most frequent. The dimension of each word is proportional to its importance and users can often find out more information about that key word via a drill-down interaction. Advanced applications include custom taxonomies of key areas for each industry, where each individual piece of feedback is automatically tagged with the key areas from the taxonomy that are present in the feedback, and the aggregation of these pieces of feedback can paint a picture of strengths and weaknesses of a company in delivering the service to customers.

Typical use cases of these insights are incorporating feedback from customers to enhance employee training and development or developing processes that amplify strengths and prevent poor customer service. For example, companies can share learnings about company strengths during new hire training to reinforce the high customer satisfaction standards. Strengths discovered in one physical retail location can be standardized across all locations. Companies can also identify the performance of specific employees or teams in order to determine whether a problem is widespread or specific to a function. This can be achieved, for example by linking CRM systems to social channels which enables the analytics software to connect the customer feedback to the team responsible for it.

\section{Competitive insights}

To be able to provide comprehensive reports that outline competitor strengths and weaknesses, online reputation management companies analyze user generated content off online review sites. The reports typically show a comparison of established metrics such as average star rating or review volume both at a physical retail location level, brand level, or at the level of a custom group of locations. Companies can understand the locations that are outperforming the competitors across the street or the locations that are lagging behind 
their competitors. A common use case is also benchmarking brands against competitor brands at a national level and using that comparison as to rally employees around the common goal of outpacing the competition.

\section{Competitive Landscape}

\section{Comprehensive benchmarks of Online Reputation}

The leader in the space is by far Reputation.com which offers an aggregated score of online reputation as well as a break-down of that score across eight different markers of brand health and perception. It is followed by Binary Fountain which offers a similar aggregated score albeit taking into account fewer drivers and only for healthcare clients. The rest of the market is mainly focused on niches, such as social reputation (Sprinklr, Hootsuite), or reputation of small and medium businesses on review sites mainly (Birdeye, Chatmeter).

\section{Digital traffic insights and analytics}

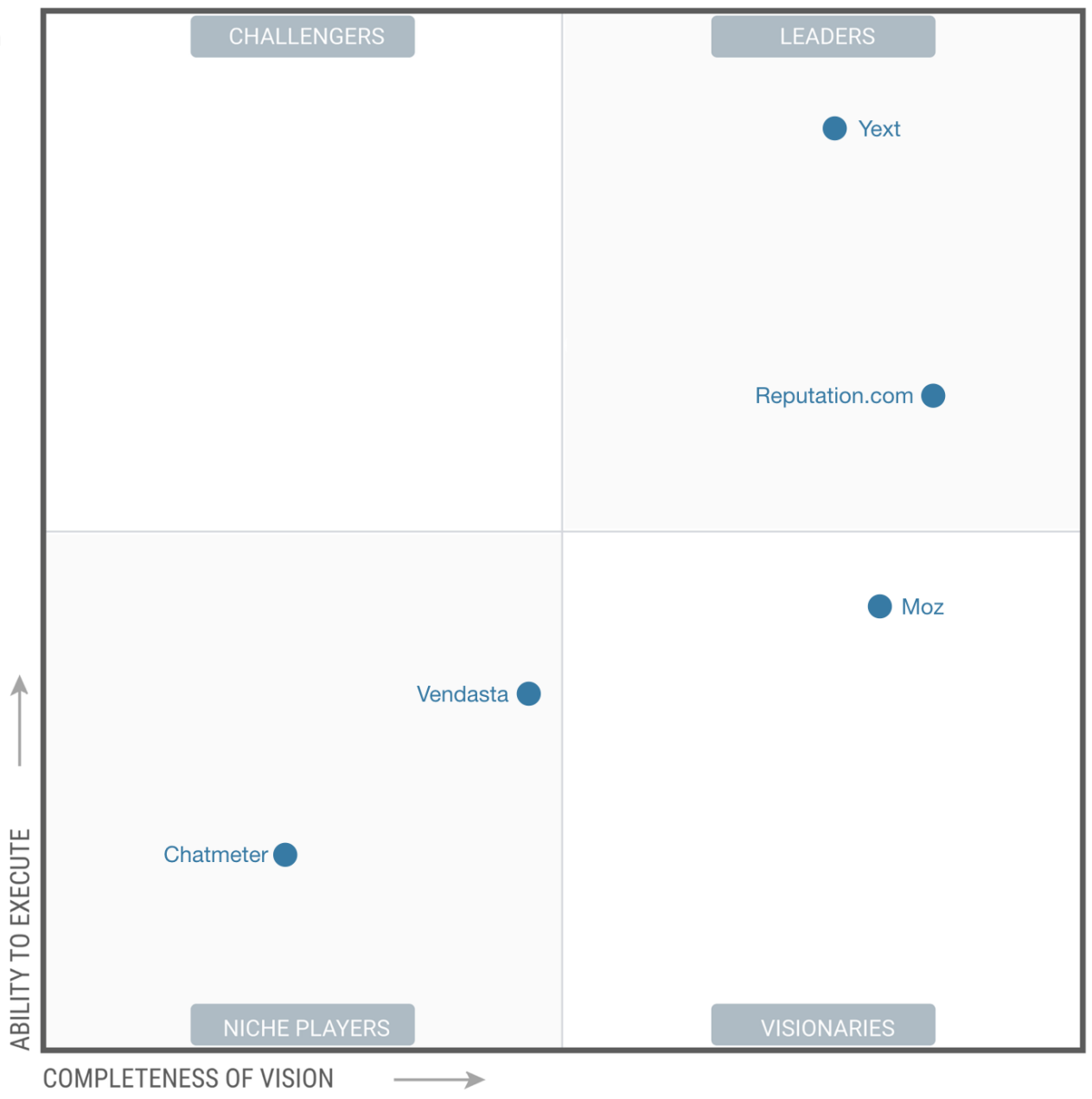

Figure 2: Traffic Insights vendors ranked using custom scoring method adapted from Gartner's

Source: Author's own research. 
Ranking criteria for Digital Traffic Insights include product capabilities such as business listings traffic analytics across multiple sources, search analytics, revenue, customer segments targeted and number of customers.

At the edge of digital traffic insights lies an area that is taking more and more mindshare of the marketing team: the online-to-offline conversion insights space. While this is an increasingly important direction of focus for many software companies, especially those in the advertising space, it is, at present, only tangential to Digital Traffic Insights, so it is not taken into consideration when evaluating vendors in the space and when comparing their existing product and solution offerings.

The most prominent sources of digital traffic insights are business listings traffic and traffic referred from search engines to digital properties of interest for businesses such as their website. Leading providers of business listings traffic have chosen different approaches to help their users extract the highest quality insights from the data. Yext, for example, has chosen a high-quantity approach: it makes a wide variety of metrics available to the user, letting them decide which are the most important for them. That can be useful for advanced users but may intimidate the majority of users. Reputation.com, on the other hand, has chosen a high-quality approach: it offers a curated report that focuses on the most important traffic insights metrics. For the expert users it also offers the ability to pick and choose insights via a reports builder feature.

While the leaders provide the ability to measure traffic insights across listings from multiple sources, such as Google My Business, Facebook, and Bing Local, the vendors focusing on SMBs, such as Vendasta only focus on Google My Business insights, and even in this single source they only provide a subset of metrics compared to the leaders. That approach is in line with the needs of small businesses, which are typically less sophisticated than those of enterprise companies, which are the representative customers of the leading vendors.

In terms of search analytics, providers such as Yext or Moz offer businesses the ability to track their search rankings, share of search by keywords, and the distribution of results depending on how consumer search to find facilities or physical retail locations. Products in this category also make comparisons possible across those metrics with local competitors.

The most sophisticated solutions for search insights take advantage of the fine grained data provided by advanced tools such as Google Analytics and Omniture. Data from these tools flow into the digital traffic insights modules, enabling businesses to understand marketing funnel conversion rates and to optimize their approach to advertising and content publishing. 


\section{Operational insights and analytics}

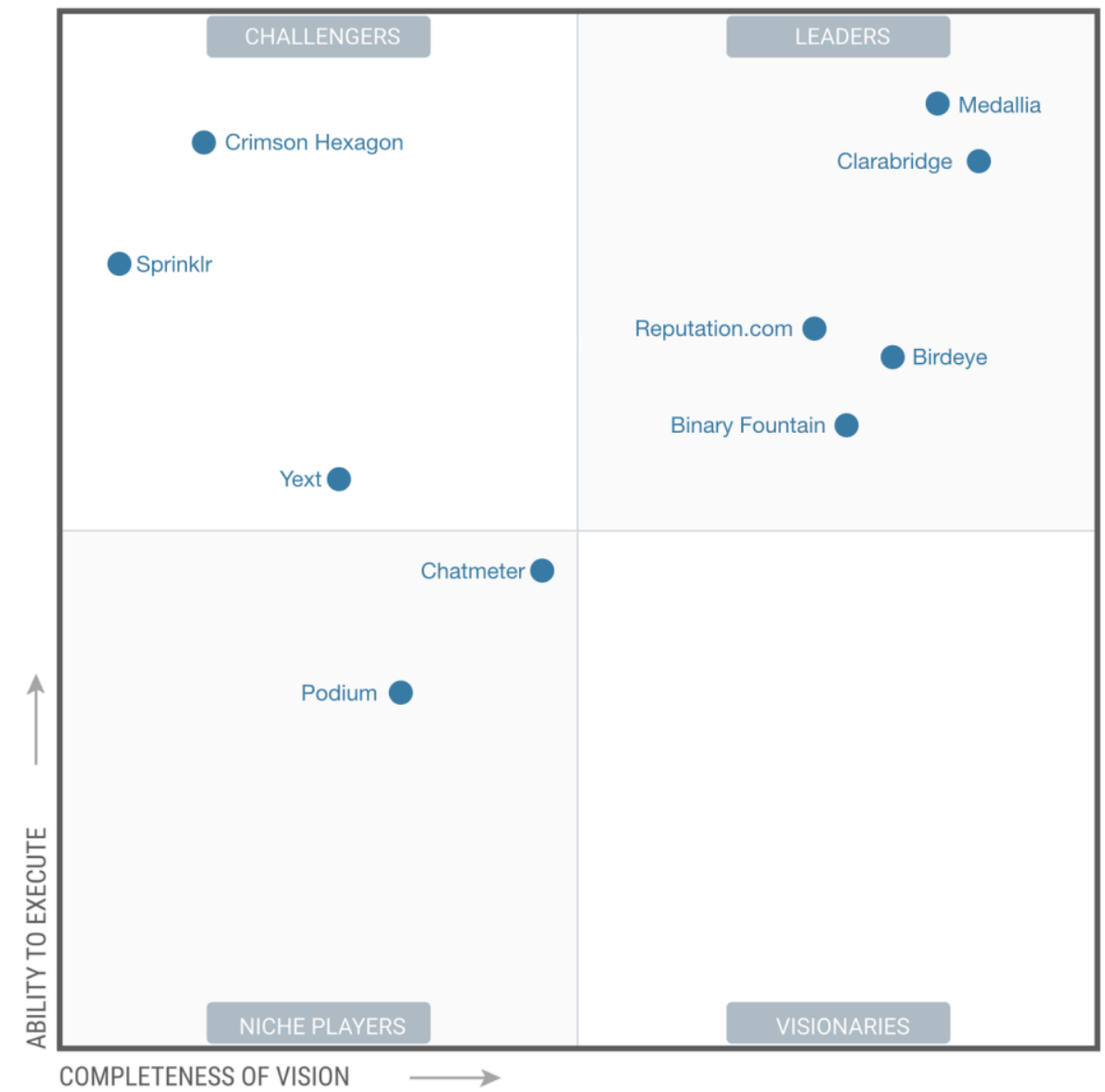

Figure 3: Operational Insights vendors ranked using custom scoring method adapted from Gartner's

Source: Author's own research.

Ranking criteria include product capabilities ranging from basic semantic analysis to advanced natural language processing and machine learning, monitored sources (review sites, custom survey data, social media, direct brand to consumer communications), industry benchmarks, revenue, customer segments targeted, and number of customers.

The operational insights space is fairly crowded, reflecting the needs of today's modern organization to derive information at scale from the unstructured customer feedback and to leverage it in making operational improvements. The established players in the customer experience space, such as Medallia, and those in the analytics space, such as Clarabridge, offer versatile solutions based on natural language processing and text analytics. They even provide statistical tools for the advanced analysis needed by data scientists that work closely with the marketing teams of client enterprises.

Other leaders such as Reputation.com or Birdeye provide robust operational insights that excel through customization options or user experience. For example, businesses can analyze their performance across topics and categories that their customers discuss across review sites, surveys, and social media, and leaders in the space enable businesses to customize the list of topics and categories they want to monitor. This ability to customize categories is very attractive for businesses wanting to assess their performance as well as 
understand their strengths and weaknesses on dimensions that are relevant to them. This feature stands in contrast with cookie cutter solutions that only offer a list of generic categories that companies can track.

Niche players in the space typically focus on a specific vertical, such as Healthcare, or target small or medium businesses. Consequently, their products are geared more towards serving the needs of the customers in those segments, which often represent subsets of those of large enterprise customers across multiple industries.

\section{Competitive insights and analytics}

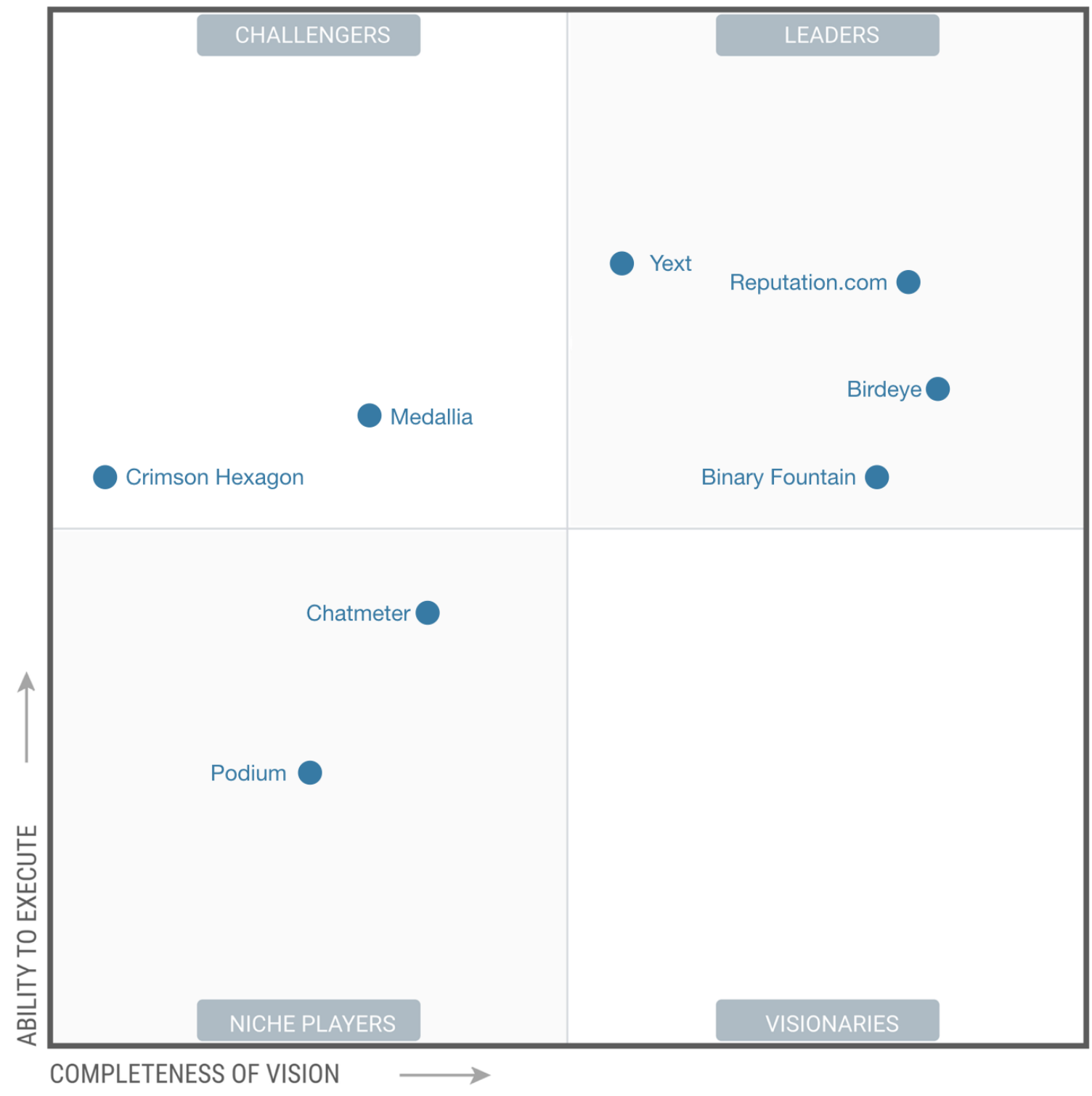

Figure4: Competitive Insights vendors ranked using custom scoring method adapted from Gartner's

Source: Author's own research.

Ranking criteria include product capabilities such as monitoring against individual competitors locally and at a brand level, custom comparisons across groups of locations, 
metrics available for comparison, competitor ranking abilities, use of text analytics for comparison, as well of speed of execution.

Some companies in the space, such as Reputation.com, provide solid and very customizable enterprise-grade solutions, while others, such as Podium or Chatmeter orient their product capabilities to the needs of small and medium businesses. Some players extract insights from a wide range of sources, while others, such as Crimson Hexagon and Sprinklr ,leverage only social media in helping their customers measure against competitors. Companies such as Birdeye and Reputation.com increasingly blend the operational insights with the competitive insights to provide their customers with a view of strengths and weaknesses across the competitive spectrum.

As a result of the different sources used for analysis by the software vendors activating in the Competitive Insights spaces, the use cases of their products also vary significantly. While some companies such as Reputation.com focus on providing a comparison revolving around online reputation, others such as Crimson Hexagon provide more niche analyses around customer segments that resonate most with Brands on social media. The wide spectrum of competitive insights provided are leveraged by different parts of various marketing organizations, and in some cases by other functions such as customer experience, or operations.

\section{Case studies}

To preserve objectivity, case studies from three different providers are included in this paper. The case studies showcase a variety of use cases and are selected from multiple industries: by getting acquainted with more types of applications in different verticals, the reader is expected to acquire a broader understanding of the space and of the possibilities that businesses have at their disposal to leverage customer experience insights to boost key performance indicators.

\section{Case study 1: Growing aggregated score of online reputation boosts auto sales}

The client was a top three US automotive manufacturer. The study tracked 1800 US auto dealerships over a six-month period.

The vendor used an aggregated score of online reputation on a 1000 point-scale as the independent variable and dealership auto sales as the dependent variable. To grow the reputation score dealerships actively requested reviews from their customers using transaction data, monitored and responded to reviews daily - engaging with clients and proactively addressing pain points in the customer experience-, they spotted opportunities of improvement using text analytics taking action to address them.

The study found that an increase by 150 points in the online reputation score led to a six percent increase in average sales, while a drop by 150 points had a 13 percent negative effect on sales. This 19 percent difference is massive considering that some analysts see the auto industry as increasingly commoditized.

\section{Case Study 2: Use of text analytics reduces churn in dissatisfied customers of telecom operator}

The client was a top two Swiss telecom carrier. The study tracked customers that expressed high dissatisfaction with service in surveys. 
The vendor used text analytics to identify at scale the customers that had a negative sentiment about the company. The algorithms detected the language associated with customer intention to terminate their contract with the mobile phone carrier. By matching surveys with customer identities, the operator call center was able to phone dissatisfied customers and attempt to appease them.

The study found that churn rate among dissatisfied customers who could be reached decreased by $40 \%$.

\section{Case Study 3: Social data informs market strategy that increases purchase intent in the coffee industry}

The client was a division selling espresso brewing machines and coffee pods of one of the top two food companies in the world. The study tracked social media for a six-month period.

The vendor tapped into a large group of coffee drinkers on social media and used natural language processing to extract valuable insights such as customer segments, their demographics and their sociographics. These discoveries allowed the client to tailor its marketing strategy and messaging to cater best to their target customers. The vendor used the same technologies and social channel to uncover feedback on the advertising developed through the marketing strategy and to adjust the ads in a way that led to better conversion. The insights software also measured the exposure of target customers to brands, additional market opportunities, and consumer intent to purchase an espresso machine.

The study found that by tailoring the marketing campaigns, product placement, and advertising, and creating a continuous improvement loop in these three categories, the client was able to drive the purchase intent over four times over the period of the study.

\section{Conclusion}

Businesses can leverage at scale the customer feedback conveyed across multiple digital channels to improve their online visibility and business operations. To be able to tap into these improvements, they need to extract actionable insights by monitoring their online reputation, analyzing their visibility on search engines, social media, and review sites, observing the key drivers of their customers' experience, and benchmarking their brands against competitors.

There are several challenges and opportunities that lie ahead for the field of customer experience analytics:

- Insights will become more nuanced, actionable and useful - by combining the different technologies and business cases: for example, businesses will be able to leverage text analytics and machine learning to understand specific strengths and weaknesses of competitors; they can then use those as inputs to strategy, operational improvements, and marketing materials that show differentiation from competitors, all which are meant to attract and retain customers. As computer learning technology improves, so will the accuracy and versatility of algorithms that classify feedback into topics and sentiments. "

- More functions will use data to make decisions - while traditionally used by marketers in order to improve their ability to attract new customers, actionable 
insights are increasingly used by other departments insights organizations in the quest for achieving improved business outcomes. Companies increasingly leverage the power of automation and data science to extract information that informs not only how to position their brands relative to competitors' or how they target prospects, but also across many other use cases: some companies leverage these insights to improve operations for existing customers, others tailor their processes or training to offer better customer service, and some others create more tailored products to their audience. It is not uncommon for insights to reach and influence the IT or Strategy departments. Managers across the entire enterprise that are relying mainly on gut feeling and intuition will become increasingly at a disadvantage to those who leverage data science and insights.

- Voice search will increase importance of insights - customers are increasingly using mobile devices and voice assistants to search for business online. Businesses will be able to use voice search analytics to identify patterns and trends and take action to improve their online presence.

- Regulation will add complexity to technical solutions - compliance with industry security standards such as HIPAA or PCI DSS and international data access regulations such as the European Union's General Data Protection Regulation will add complexity to software solutions that extract insights from large amounts of unstructured data.

- Customers will trade off privacy for better customer experience - as businesses integrate CRM data, transaction data, online traffic data, demographic data and customer preferences, they will use artificial intelligence to create a more complete profile of the consumer, and to delight them with a better experience. Businesses need to approach the increasing amounts of consumer data in an ethical manner and to give consumers the ability to protect their privacy.

\section{References}

Magic Quadrant (2018) retrieved at https://en.wikipedia.org/wiki/Magic_Quadrant Higher Reputation Score Boosts Auto Sales (2018) retrieved at https://www.reputation.com/resources/case-study/better-online-reviews-boostauto-sales/

Psarouthakis, J. (2010), The Auto Industry is Now a Commodity Business retrieved at http://businessthinker.com/the-auto-industry-is-now-a-commodity-business/

Dodani, S, Kunzi, U. (2017) - Transforming Your Business with Unstructured Customer Feedback (featuring Sunrise Communications) retrieved at https://vimeo.com/medallia/review/241797789/06d38cf65c

Optimize Customer Experience with insights from online reviews (2018) retrieved at https://www.reputation.com/resources/white-paper/optimize-customerexperience-with-insights-from-online-reviews/

Sprinklr Benchmarking Insights (2018) retrieved at https://www.sprinklr.com/benchmarking-insights/

Moz (2018) retrieved at https://en.wikipedia.org/wiki/Moz_(marketing_software) Vendasta introduces Google My Business Insights into Listing Builder providing actionable customer insights to local businesses (2017) retrieved at 
https://www.vendasta.com/blog/vendasta-introduces-google-business-insightslisting-builder-providing-actionable-customer-insights-local-businesses

How to Grow ARR 48\% YoY from $\$ 17 \mathrm{~m}$ to $\$ 28 \mathrm{~m}$ in SMB SaaS Space (2018) retrieved at http://nathanlatka.com/thetop925/

Birdeye Business Insights (2018) retrieved at https://birdeye.com/business-insights/

Winter '18 Release Available Now: Get Early Access to the Yext Knowledge Assistant (2018)

PICBE | 815 retrieved at https://www.yext.com/blog/2018/01/winter-18-release-early-accessto-the-yext-knowledge-assistant/

Using Social Insights to Build a Global Marketing Strategy - Nespresso (2015) retrieved at http://pages.crimsonhexagon.com/rs/284-XQB-702/images/Using-Social-Insightsto-Build-a-Global-Marketing-Strategy-Nespresso.pdf

How Social Intelligence can guide decisions (2018) retrieved at https://www.mckinsey.com/industries/high-tech/our-insights/how-socialintelligence-can-guide-decisions 